\title{
Interfaces
}

\section{Les vitraux pour Jérusalem de Chagall : expositions et réception}

\section{Christel Scheftsik Naujoks}

\section{(2) OpenEdition}

1 Journals

Édition électronique

URL : http://journals.openedition.org/interfaces/649

DOI : 10.4000/interfaces.649

ISSN : 2647-6754

Éditeur :

Université de Bourgogne, Université de Paris, College of the Holy Cross

\section{Édition imprimée}

Date de publication : 21 juin 2019

Pagination : 101-116

ISSN : 1164-6225

\section{Référence électronique}

Christel Scheftsik Naujoks, "Les vitraux pour Jérusalem de Chagall : expositions et réception», Interfaces [En ligne], 41 | 2019, mis en ligne le 21 juin 2019, consulté le 06 janvier 2021. URL : http:// journals.openedition.org/interfaces/649; DOI : https://doi.org/10.4000/interfaces.649

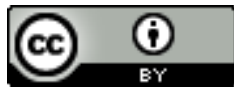

Les contenus de la revue Interfaces sont mis à disposition selon les termes de la Licence Creative Commons Attribution 4.0 International. 


\title{
LES VITRAUX POUR JÉRUSALEM DE CHAGALL : EXPOSITIONS ET RÉCEPTION
}

\author{
Christel Scheftsik Naujoks \\ EA 7347 Histara, École Pratique des Hautes Études, Paris ; \\ Centre des Sciences Historiques de la Culture, Université de Lausanne, Suisse
}

Durant les deux années 1960 et 1961, Chagall réalise douze vitraux pour la synagogue du Centre hospitalier Hadassah à Jérusalem. À cette date, le peintre judéo-russe a déjà conçu deux vitraux pour la chapelle d'Assy et certains vitraux pour la cathédrale de Metz. Il interrompt le projet messin pour se consacrer entièrement aux vitraux pour Jérusalem ; il y reviendra immédiatement après le chantier israélien. Peu étudiés dans l'historiographie de l'art du vitrail moderne, ce programme a été essentiellement commenté par la communauté juive, et pour cause, les douze vitraux pour la synagogue du centre Hadassah sont les seuls que Chagall ait créés pour un lieu de culte juif. S'il a surtout eu l'occasion de réaliser des vitraux pour des cathédrales, il n'en est pas moins resté fidèle au judaïsme de son enfance et de ses ancêtres, quoiqu'il ne soit pas pratiquant ( Je ne peux pas prier. Je travaille seulement $»^{1}$ ), et que les emprunts à l'iconographie chrétienne dans son œuvre fassent polémique auprès de certains de ses co-religionnaires.

C'est à l'occasion de la grande rétrospective parisienne de l'œuvre du peintre au Louvre en juin 1959, présentant justement un vitrail, celui de Jérémie, prévu pour la cathédrale de Metz, que Miriam Freund, présidente de la société Hadassah et l'architecte Joseph Neufeld décident de passer commande à Chagall de vitraux pour une petite synagogue en cours de construction. La société Hadassah est une organisation sioniste américaine de bienfaisance, fondée en 1912, qui est à l'origine, notamment, de la construction de l'ensemble du centre médical de l'université hébraïque Hadassah et de sa synagogue à Ein Karem, près de Jérusalem.

Les vitraux forment une couronne au-dessus de la bimah², à raison de trois vitraux sur chacun des quatre côtés, se conformant ainsi à la recommandation du Zohar de « faire entrer un maximum de lumière dans la synagogue et pour cela de faire percer douze fenêtres » (Jarrassé, Une Histoire des

«Entretien avec Chagall $»(84)$.

La bimah (pour les juifs ashkénazes - ou tebah pour les séfarades) est l'estrade équipée d'une table sur laquelle les rouleaux de la Torah sont posés pour être lus pendant l'office. La bimah se situe généralement au centre de la synagogue 
synagogues françaises 352). Le programme iconographique est tiré des deux textes vétérotestamentaires de la Genèse ${ }^{3}$ et du Deutéronome ${ }^{4}$, chaque fenêtre se rapportant à l'une des douze tribus d'Israël, soit aux douze fils de Jacob ${ }^{5}$. Ce thème des douze tribus est largement représenté dans l'art synagogal, en raison de la multiplicité des textes bibliques et midrachiques qui s'y réfèrent comme par la profusion des références au nombre douze ; «L'importance du thème des douze tribus semble bien liée à la représentation du peuple dont la synagogue est le lieu de rassemblement » (Jarrassé, Une Histoire des synagogues françaises 351). Si le thème et la disposition par trois sont des caractéristiques connues, notamment à la synagogue de la rue de la Victoire à Paris, Chagall a pris quelques libertés. En effet, plusieurs choix plastiques prévalent sur le texte biblique : c'est en peintre que Chagall associe une couleur de son choix (le rouge, le vert, le jaune ou le bleu) à chaque tribu, c'est encore en artiste qu'il décide de l'emplacement et de l'ordre de chaque vitrail - la disposition respectant « le schéma prosodique aam [...] typique des livres prophétiques » (Harshav, « Les vitraux de Jérusalem à la lumière de la poétique chagallienne »126) est néanmoins respecté. Rivon Krygier suggère que « la distribution des vitraux dans la synagogue de l'hôpital Hadassah [...] se rapporte à la répartition des tribus, dans le campement du désert tout autour du Tabernacle » (21). Respectueux de l'interdit de la représentation humaine, Chagall développe un bestiaire et un ensemble de symboles pour désigner chaque fils de Jacob. Il choisit de conserver la tribu de Lévi, au détriment de celles d'Ephraïm et Manassé, les fils de Joseph, suivant la Genèse, plutôt que le Deutéronome. Ainsi, « la structure amphictyonique adoptées dans la disposition des vitraux doit alors être entendue comme une simple allusion à la structure du campement dans le désert, tout en se référant directement à la filiation directe de Jacob dans laquelle Lévi occupe naturellement sa place, parmi les autres frères » (21).

Notre propos consiste ici à examiner trois moments muséographiques : l'exposition des douze vitraux d'abord à Paris au musée des Arts Décoratifs à l'été $1961^{6}$, puis à New York au Museum of Modern Art à l'automne de la même année ${ }^{7}$, enfin leur installation définitive dans la synagogue du Centre Hadassah à Jérusalem ${ }^{8}$. Ces trois évènements muséographiques doivent être étudiés d'une

conformément à la disposition dans le Temple de Jérusalem, mais les synagogues réformées, sur le modèle des églises, placent volontiers la bimah au fond de la synagogue, côté est.

Genèse 49 (les douze bénédictions de Jacob avant de mourir).

Deutéronome 33 (La bénédiction de Moïse à la veille de sa mort).

Ruben, Siméon, Lévi, Juda, Zabulon, Issachar, Dan, Gad, Asher, Nephtali, Joseph, Benjamin.

Prévue initialement du 16 juin au 30 septembre, l'exposition fut prolongée de deux semaines.

Du 19 novembre 1961 au 3 janvier 1962.

Inaugurée le 6 février 1962. 
part pour eux-mêmes (commande, scénographie), d'autre part pour leur réception par la critique d'art contemporaine.

En effet, l'exposition, avant scellage, de vitraux dans des musées est une singularité propre à Chagall ; sauf erreur de notre part, seuls les vitraux de Chagall ont été exposés temporairement en musée avant scellage définitif dans un édifice religieux. Cette singularité est doublement notable : par leur nature monumentale, les vitraux ne sont pas des objets d'expositions temporaires, à plus forte raison itinérantes, et par leur vocation première à décorer un lieu de culte, les vitraux ne sont pas, par nature, des objets d'art comme les autres, a fortiori à partir du moment où ils sont exposés dans un musée. Dès lors que des vitraux prévus pour un lieu de culte sont exposés dans un musée, qu'ils sont de ce fait décontextualisés, deux questions émergent : assiste-t-on à une modification sémantique des vitraux ? Cette modification sémantique impacte-t-elle l'image que l'on se fait de Chagall ? Par modification sémantique, nous entendons le glissement de sens, l'altération de la compréhension d'une œuvre, soumise à un autre processus, celui de la représentation - et de l'interprétation, c'està-dire la manière dont les historiens ou critiques d'art pensent une œuvre et en parlent à partir de leur subjectivité. En cela, cette étude rejoint la notion de représentation telle que Chartier l'a exposée et s'inscrit dans une histoire de la réception de l'œuvre de Chagall, permettant par cette voie de mettre à l'honneur l'étude de l'artisanat d'art, celui du vitrail en particulier. Rappelons qu'un grand nombre de revues anglo-saxonnes et françaises ${ }^{9}$, spécialisées ou non, publient des articles consacrés à l'art juif, et à Chagall, en particulier au sujet de ses vitraux et des numéros spéciaux complets sont consacrés à Chagall à l'occasion de l'exposition parisienne ${ }^{10}$. En somme, interroger la réception en ce début des années 1960 des douze vitraux de Chagall présente un intérêt double dans la mesure où cela contribue à la recherche en muséographie et historiographe de l'art juif moderne comme à celle sur l'artisanat d'art dans l'histoire de l'art : l'étude de l'art juif en général comme champ de recherche en histoire de l'art est en soi très récente ; une première phase de publications se développe dans les années 1970, mais c'est seulement depuis une vingtaine d'années que les recherches sur l'art juif s'intensifient, situant généralement Chagall tantôt au sommet de l'art juif moderne, qui, depuis l'Émancipation, peut enfin émerger, tantôt en tant que dernier représentant d'un art juif désormais voué à s'éteindre.

Les expositions parisienne et newyorkaise sont l'occasion de montrer les vitraux avant leur scellage définitif à Jérusalem, mais aussi d'en exposer la genèse par ses étapes successives, dessins

The Chicago Jewish Forum, The Canadian Jewish Chronicle, Jewish Spectator, Art Journal, A.J.A. Quarterly, The Progressive, Jewish Frontier, Congress bi-weekly, The Art Journal, Molad, Studies in Art, Scripta Hierosolymitana, Bulletin de nos Communautés.

10. Notamment, la revue L'Arche, côté juif, et revue L'Art Sacré, côté chrétien. 
préparatoires, croquis, maquettes et panneaux d'essai. L'exposition parisienne s'inscrit dans les deux décennies, entre 1945 et $1965^{11}$, durant lesquelles le mécénat étatique français est très actif. Depuis le 8 janvier 1959, André Malraux est le ministre d'État des affaires culturelles du gouvernement de Michel Debré sous la présidence de Charles de Gaulle. La commande des vitraux pour Metz (dont le projet débute en 1958), délaissés par Chagall au profit de ceux pour Jérusalem, peut être considérée comme la première commande de l'État français puisque c'est Robert Renard, architecte en Chef des Monuments Historiques de 1946 à 1974, qui prend l'initiative de ce projet. Toutefois, c'est sans consulter le ministère de la Culture qu'il choisit le peintre judéo-russe, après avoir déjà passé commande à Villon, puis Bissière. Le projet Chagall pour la cathédrale de Metz trainait, les prétextes étant multiples, entre autres l'idée selon laquelle il était impossible qu'un artiste au goût si prononcé pour le folklore russe œuvre pour une cathédrale gothique ; il aura fallu un « coup de pouce » de Malraux pour que le projet se concrétise. C'est encore son ami Malraux, conscient de « l'intérêt majeur d'une des réalisations les plus achevées du grand artiste qu'est Chagall » (Chagall. Vitraux pour Jérusalem 8), qui est moteur dans l'organisation de l'exposition parisienne. Ces nombreuses collaborations sont par ailleurs le fruit d'une durable et sincère amitié entre Malraux et Chagall qui remonte à presque quatre décennies ${ }^{12}$.

L'exposition parisienne des vitraux a lieu dans « un bâtiment léger adhérent au Pavillon de Marsan comme une moule à la coque d'un navire » (Bourdet 141); il s'agit d'une structure provisoire, construite par Marcel Roux, en sus des bâtiments historiques du palais des Tuileries. Roger-Marx souligne qu' « une lumière d'en haut, qui n'est pas seulement celle du soleil, une lumière à la fois extérieure et intérieure, donne à ces grandes compositions leur résonance et leur splendeur » (157). Charensol apprécie ces choix en matière d'éclairage naturel :

« Ces problèmes d'éclairage [...] ont trouvé une solution d'une remarquable élégance au jardin des Tuileries où un pavillon attenant au Musée des Arts Décoratifs nous montre les douze immenses verrières [...]. Discrètement dissimulé en bordure du Carrousel, ce pavillon se présente comme une galerie sinueuse uniquement conçue pour présenter à la lumière du jour ces vitraux aux couleurs éclatantes » (484).

11. 1965 marque le début de la décentralisation artistique, c'est-à-dire la période où l'État cesse d'être le seul commanditaire des artistes (voir à ce sujet le catalogue d'exposition Un art d'État? 2017).

12. C'est un jeune Marc Chagall (1887-1985) fraîchement de retour à Paris après l'appel de Cendrars, et cette fois avec sa femme Bella et sa fille Ida, qu'André Malraux (1901-1976) rencontre pour la première fois en 1924. L'écrivain, lui, revient de son premier et tumultueux voyage en Indochine. Il visite la première exposition parisienne du peintre judéo-russe à la galerie Barbazange-Hodebert. Leur amitié ne sera interrompue que par la mort de Malraux en 1976. La relation entre les deux hommes et son impact sur la réception de l'artiste a fait l'objet d'une communication par nos soins, dans le cadre des journées d'étude « Art et Pouvoir : le pouvoir de l'art » (EPHE-INHA, le 26 octobre 2017). Un article issu de la communication est à paraître prochainement. 
La scénographie parisienne privilégie donc la monstration des vitraux en longueur, côte à côte, comme un développement de panneaux formant une « galerie sinueuse $\rangle^{13}$. À Paris, comme à New York, ils sont placés à hauteur d'homme, ce qui est un choix de Chagall ${ }^{14}$, alors qu'ils sont en hauteur dans la synagogue. Mais contrairement à celle de Paris, l'exposition newyorkaise n'est pas isolée du reste des œuvres du musée ${ }^{15}$. Les vitraux y sont exposés dans des arches spécialement conçues à l'identique de celles dans lesquelles ils seront insérés dans la synagogue. L'ordre des vitraux, décidé par Chagall pour la synagogue est repris dans l'exposition ${ }^{16}$. L'éclairage des vitraux se fait par des rangées de tubes fluorescents placés derrière les vitraux, palliant à l'impossibilité d'un éclairage naturel. À New York résident une importante communauté juive, dont les membres de la société Hadassah, mécènes des vitraux pour Jérusalem. Dès lors, il convenait de leur présenter l'ensemble, avant le départ des vitraux pour Israël.

La récurrence d'expositions temporaires et/ou itinérantes d'objets d'art monumentaux demeure une singularité muséographique propre à l'œuvre de Chagall : si une dizaine d'expositions entre 1959 et 1970 montre en musée des vitraux avant leur scellage définitif dans l'édifice religieux de destination, seule l'exposition parisienne expose un cycle complet de vitraux. Compte tenu de cette singularité, il est intéressant d'observer dans quelle mesure l'exposition d'objets d'art cultuels prévus effectivement pour un lieu de culte, dont le thème est biblique, réalisés par un artiste juif, mais exposés dans des musées nationaux, avec tous les attributs qu'implique une telle exposition (catalogue d'exposition, publicité,

13. Une photographie de la scénographie est visible dans le catalogue d'exposition Marc Chagall. Hadassah. From Sketch to Stained Glass Windows, à l'occasion de l'exposition Marc Chagall. Hadassah, de l'esquisse au vitrail, qui s'est tenue au Musée d'Art et d'Histoire du Judaïsme (30 avril - 15 septembre 2002 : illustration n7 (141).

14. Lettre Chagall-Marq, 13 septembre 1961 : « I think the exhibition of stained glass windows that is closing here [in Paris] at the end of September will leave for the Museum of Modern Art in New York shortly after. But I am worried about the windows. I do not want that ensemble to be theatrical. If a special wing cannot be created, they should at least be exhibitioned as paintings [sic], not very high » (cité par Harshav, Chagall and His Times 885).

15. «And Redon whose works were shown in the Museum of Modern Art along with Chagall's windows [...]» (Werner, « Chagall's Jerusalem windows » 542). Réédité en 1971 dans un recueil d'essais par Gutmann dans No Graven images: Studies in Art and the Hebrew Bible, édité à New York). Alfred Werner (1911-1979). Historien de l'art et journaliste austro-américain. Libéré de Dachau en 1939, s'enfuit aux États-Unis, a abondamment contribué à des revues d'art comme à des revues juives, en écrivant sur la politique européenne et le sionisme, l'art européen et américain des $\mathrm{XIX}^{\text {ème }}$ et $\mathrm{XX}^{\text {ème }}$ siècles, et plus particulièrement sur les artistes juifs et israéliens.

16. « Museum installation [sic] closely follows Chagall's own design for their placement in the synagogue » (Peter Selz, conservateur au département de peinture et de sculpture du MOMA et commissaire de l'exposition, dossier de presse de l'exposition de New York). Des photographies de l'exposition sont visibles sur le site du MoMA : https://www. moma.org/calendar/exhibitions/3421?locale=fr (page consultée le 20 mai 2019). 
entrée payante, visibilité temporaire des œuvres, etc.) modifie la portée sémantique de l'oeuvre, et le regard sur l'artiste.

Il apparaît dans les discours écrits que le lieu et la scénographie sont deux paramètres largement décrits dans la presse faisant état de ces vitraux, de leur exposition parisienne ou newyorkaise comme de leur inauguration à Jérusalem, et ce, soit dans une simple démarche descriptive, soit, précisément, pour en discuter la portée.

Prévus pour une synagogue, les douze vitraux des Tribus d'Israël, par la technique (l'art du vitrail est historiquement un art religieux), par le thème (la fondation d'Israël), par l'emplacement (un édifice religieux), par le créateur (un artiste juif), possèdent indéniablement toutes les caractéristiques de l'objet d'art religieux. Pourtant, et dès l'origine du projet, s'opèrent des modifications sémantiques. Équiper un centre hospitalier d'un lieu de culte est, de prime abord, un choix fonctionnel ; il revêt pourtant immédiatement une nouvelle signification artistique, symbolique, même politique. En 1959, l'État d'Israël n'a que onze ans et le judaïsme renaît de ses cendres ; on construit des synagogues autant qu'on reconstruit le judaïsme. Et si l'architecture religieuse juive est marquée par un renouveau artistique, ce n'est pas sans lien avec le renouveau artistique qui occupe les cercles chrétiens, avec, en chef de fil, le père Marie-Alain Couturier. C'est au côté de Maurice Denis et Georges Desvallières, dans les Ateliers d'Arts Sacrés, que Pierre Couturier (1897-1954), peintre et verrier, étudie l'art dès 1919. Celui-ci éprouve très vite de l'admiration pour les grands maîtres de la peinture moderne. Dès lors, celui qui est devenu le Père Marie-Alain Couturier de l'Ordre des Frères Prêcheurs en 1925 va prendre l'initiative de confier la décoration de la chapelle Notre-Dame-de-Toute-Grâce d'Assy (Haute-Savoie) à des artistes modernes. L'église du Plateau d'Assy, commencée en 1938, est consacrée le 4 août 1950. Construite par l'architecte Maurice Novarina, elle est située à 1200 mètres d'altitude et devait permettre aux curistes et aux personnels sédentaires des sanatoriums du plateau de bénéficier d'un véritable lieu de culte. Le chanoine Devemy et le Père Couturier font notamment appel à Fernand Léger, qui réalise la mosaïque de la façade sur le thème des litanies de la Vierge, et Jean Lurçat, chargé de la tapisserie du maître-autel ; à Lipchitz et Chagall sont confiées respectivement la réalisation d'une sculpture de la Vierge et la décoration du baptistère ${ }^{17}$. Matisse et Bonnard réalisent chacun un dessus d'autel latéral. Rouault, Bazaine, Signori, Braque, Germaine Richier, entres autres, collaborent également à la décoration de l'église. Les artistes reçoivent une totale liberté de création, quoiqu'ils doivent s'adapter à l'architecture de l'édifice. Chacun y met sa « personnalité propre, au risque de scandaliser le clergé en porte-à-faux avec la modernité proposée » (Bouvier et Cousin 32). Deux débats émergent donc de ce projet : la place de l'art abstrait dans l'art cultuel et la participation des artistes modernes dans la 
construction et la décoration d'édifices religieux. Artistes et architectes de renom (tels que Breuer, Matisse, Le Corbusier, Niemeyer, Wright, etc.) construisent dans leur globalité, dès les années 1950, des édifices religieux, en en concevant tous les composants, de l'architecture aux objets liturgiques en passant par le mobilier.

Joseph Neufeld (1899-1980), lui, a étudié l'architecture à Vienne puis à Rome, et a travaillé à Berlin à la fin des années 1930 avec Erich Mendelsohn, puis jusqu'en 1933 avec Bruno Taut à Berlin et Moscou. Après avoir fait son alyah en 1920, il est l'un des trois architectes qui constituent le noyau d'origine du Cercle des Architectes fondé en 1932 ; avec Arieh Sharon et Ze'ev Rechter, il s'agit de réfléchir aux modalités d'un style architectural propre aux Juifs d'Israël. La réponse de Neufeld tient en deux mots : organisme (en tant que perfection des formes issues de la nature) et rationalité. Fonctionnalité et économie des lignes seront leurs priorités, héritées du Bauhaus. Neufeld dessine notamment le début de la rue Allenby à Tel Aviv, des résidences ouvrières, le siège de la Koupat-Holim, etc., essentiellement des projets publics. En 1940 c'est en tant qu'architecte renommé que Neufeld émigre aux États-Unis. En 1949, il reçoit la commande pour le Centre hospitalier universitaire Hadassah près de Jérusalem.

Dix ans plus tard, lorsqu'il visite avec Miriam Freund la rétrospective Chagall au musée des arts décoratifs à Paris, le peintre est lui aussi un artiste majeur du paysage artistique moderne; pourtant c'est malgré la persistance de la figuration et la récurrence des thèmes bibliques dans son œuvre que Chagall a su s'imposer, - et en même temps demeurer en marge des mouvements d'avant-garde : le Louvre lui consacre une rétrospective, et l'État lui commande des vitraux pour ses cathédrales.

Sans conteste, durant les quatre premières années de la décennie 1960 se produit un bouleversement majeur dans la réception de l'œuvre de Chagall : de la commande des vitraux pour Jérusalem à l'inauguration du plafond de l'opéra commandé par Malraux, la place désormais incontestable de Chagall parmi les maîtres de l'art moderne sera l'occasion pour la communauté juive de se réapproprier l'artiste juif. En effet, " la communauté juive recevait assez mal son activité dans les églises » (Jarrassé, « Remarques sur la symbolique des vitraux de Jérusalem » 46). Miriam Freund tenait là la bonne occasion « de mettre le peintre juif en situation de glorifier le Judaïsme » (46). En effet une bonne partie de la communauté juive n'hésitait pas à vilipendait Chagall en raison de ses vitraux pour des édifices chrétiens, comme elle le faisait déjà à cause de la récurrence du Christ crucifié dans ses tableaux. Chagall en était d'ailleurs suffisamment troublé pour demander conseil aux Juifs « ayant la tête sur les épaules $»^{18}$. La commande pour un édifice juif était donc un événement très attendu : « Le 
voilà enfin qui vient à une synagogue... ${ }^{19}{ }^{19}$. Et paradoxalement, malgré l'interdit de l'image du Second Commandement, on considéra que la décoration d'une synagogue à Jérusalem lui offrirait l'opportunité de s'exprimer enfin librement ${ }^{20}$. Dans ce processus d'appropriation par la communauté juive du cycle des douze vitraux pour la synagogue, apparaît une salutaire interférence de la communauté juive avec la renommée de Chagall. L'exposition des vitraux en contexte muséal s'inscrit dans une période de généralisation des expositions d'art juif à partir de 1950. Le contexte matériel et financier est en effet propice à cette éclosion muséographique. «L'amélioration des conditions matériels de toutes ces communautés au cours des années 50 se traduit par un essor de l'activité caritative, qui devient l'un des axes majeurs de la vie communautaire » (Wasserstein 100). Au cours des années 1960, cette dynamique ira croissant avec une forte augmentation du nombre d'expositions consacrées à l'art juif. Cela s'explique par le changement de vision des Juifs sur eux-mêmes. Avec la création de l'État d'Israël, « l'homme juif nouveau est en train de naître sous le regard médusé des nations. A l'image du vieux yid du ghetto au teint blafard, à celle du déporté décharné, se substitue celle du sabra, un homme dans la force de la jeunesse, robuste et musclé » (Azria 20). Régine Azria, qui a étudié la réception du Juif par les Juifs et les non-Juifs, présente comment, à partir des années 1960, émergent un nouvel aspect du judaïsme qui tente, non plus de se formater aux normes de la modernité, mais au contraire d'affirmer le particularisme juif (22). Avec la commande des vitraux pour la synagogue, la communauté juive ne cherche pas à s'extraire de son particularisme juif, mais à rappeler au monde la judaïté de l'artiste. Et de même que la question de l'existence d'un art juif s'est superposée à la question de l'identité juive dans les débats des années 1920, de même la question identitaire juive qui ressurgit après guerre et induit un nouvel élan architectural et muséographique, suscite immanquablement le retour de la question de l'existence d'un art juif. ${ }^{21}$ Cette à cette question que Miriam Freund propose une réponse. Ainsi, la décision de passer commande à Chagall pour des vitraux transforma significativement la simple valeur fonctionnelle de la synagogue en un manifeste pour un art juif moderne en terre d'Israël. Les vitraux de Chagall dans la synagogue de Neufeld prennent des airs de porte-drapeau d'une modernité artistique spécifiquement juive.

19. Roger Berg, Bulletin de nos Communautés 13 (7 juillet 1961) cité par Jarrassé (« Remarques sur la symbolique des vitraux de Jérusalem » 47).

20. «En 1957 et 1958, Chagall réalise des vitraux et des maquettes de vitraux pour une église en Savoie et pour la cathédrale de Metz. Mais, là où il peut, en vérité, 'se laisser aller' [l'auteur reprend les mots de Chagall qu'il cite déjà précédemment dans son article] [...], c'est dans les douze grands vitraux pour Jérusalem [...]» (Baram 106).

21. Lire à ce sujet l'analyse que fait Dominique Jarrassé du numéro spécial que consacre en 1961 la revue $L$ 'Arche à la question « Existe-t-il un art juif? » (Jarrassé, Existe-t-il un art juif? 2006). 
De surcroît, si le christianisme n'a pas pu traduire en son langage propre le thème des douze tribus, mais seulement du nombre douze (Jarrassé, Une Histoire des synagogues françaises 356), « on comprend [...] que les juifs aient pu user des représentations des tribus, image du peuple juif, dans un but identitaire, et soient parvenus, tout en recourant à ce thème demeuré conventionnel dans le cadre religieux, à lui insuffler une valeur culturelle spécifique. L'architecture et le décor sont alors le support d'un message d'unité et de solidarité par-delà la diversité des composantes du judaïsme contemporain » (356-57). Et, selon Miriam Freund, unité et solidarité sont désormais possibles en Israël. Née à New York d'une mère qui appartenait au premier groupe de femmes sionistes de la côte Est (les Filles de Sion), Miriam Freund (1906-1999) était imprégnée dès l'enfance des discussions des cercles d'intellectuels juifs américains favorables au sionisme. Proche de Henrietta Szold, pionnière et fondatrice de l'organisation de femmes sionistes d'Amérique, la Hadassah, elle est invitée en 1940 à rejoindre le Conseil national de la Hadassah, où elle occupera plusieurs fonctions.

Selon Chartier, « la notion de représentation [...] permet d'articuler trois registres de réalités : d'une part, les représentations collectives qui incorporent dans les individus les divisions du monde social et qui organisent les schèmes de perception à partir desquels ils classent, jugent et agissent ; d'autre part, les formes d'exhibition et de stylisation de l'identité qu'ils entendent voir reconnue ; enfin, la délégation à des représentations (individus particuliers, institutions, instances abstraites) de la cohérence et de la stabilité de l'identité ainsi affirmée. L'histoire de la construction des identités sociales se trouve ainsi muée en une histoire des rapports de force symboliques » (11-12). C'est en partie dans ce troisième registre de la représentation que se situe la synagogue du centre Hadassah. Mais Chagall n'est pas l'unique médium de cette identité affirmée : Le choix artistique de commander des vitraux, - peut-être celui de Neufeld, qui, en héritier du Bauhaus, cherche à réhabiliter l'artisanat et à produire un art global - comme le choix iconographique des douze tribus et celui de passer commande à Marc Chagall contribuent donc simultanément et sans pouvoir être dissociés à superposer les trois significations sociale, politique et artistique de cette nouvelle synagogue dans un Israël en plein essor.

Cette modification sémantique des vitraux, désormais compris comme une partie d'un tout à portée symbolique dépassant le cadre artistique, se confirme rapidement par leur succès même. Dès 1962, Alfred Werner, dans son article sur les vitraux de Chagall pour Jérusalem dans Art Journal, met en garde contre les risques liés à une mauvaise compréhension de l'artiste, et donc de l'œuvre : après une mise au point quant à la différence sémantique entre des vitraux vus dans une église et ceux d'une synagogue, écartant d'emblée toute tentative de supplémenter une valeur liturgique aux vitraux de Chagall, il rapporte que, si dans le passé les gens ne critiquaient pas ouvertement l'artiste réputé, c'était par respect pour les sacro-saints noms tel que Picasso, Matisse ou Chagall. À l'heure où il écrit, 
Werner espère que c'est en connaissance de cause que l'œuvre du peintre judéo-russe est admirée ${ }^{22}$. De ce fait, Werner interroge la manière de considérer la synagogue ainsi décorée par les vitraux d'un de ces « sacro-saints noms » de l'art moderne : «temple de l'art» ou « temple de Dieu » ${ }^{23}$. Selon Werner, la modification sémantique, du cultuel au culturel, s'opère à partir du moment où les œuvres, réalisées par un artiste reconnu comme l'un des maîtres de la peinture du $\mathrm{XX}^{\text {ème }}$ siècle, s'enchâssent dans les murs de l'édifice. C'est alors la synagogue, qui, en recevant les vitraux de Chagall, tend à se métamorphoser ${ }^{24}$ en musée. Werner achève son argumentaire en montrant les limites mêmes de la réalisation de la décoration d'un lieu de culte par un artiste moderne en donnant son avis sur la chapelle Matisse à Vence, laquelle lui semble davantage s'adresser aux amateurs d'art agnostiques qu'elle ne permet d'entrer en communion avec Dieu. Le fait de passer commande à un artiste moderne d'œuvres prévues pour un lieu de culte remet en question la nature même de ce lieu de culte, glissement qui s'opère alors en faveur d'une compréhension muséale du lieu.

Jean Leymarie, dans son ouvrage sur les vitraux pour Jérusalem paru cette même année 1962, formule lui aussi une réserve sur le mélange des genres sacré/moderne. Sa retenue concerne l'église d'Assy qu'il qualifie d' " expérience artificielle et disparate ${ }^{25}$. Quoique il en reconnaisse le caractère unique et historique, il affirme avec bienveillance qu'au contraire d'Assy, les vitraux pour Jérusalem font figure de « monument artistique souverain et message religieux vraiment universel qu'aucune limitation confessionnelle ne restreint $[\ldots] »^{26}$.

Werner va néanmoins mettre en garde Jean Leymarie contre une analyse des vitraux en tant que pièces de musée, ex situ, feignant même de supposer qu'il n'a pas vu les œuvres scellées dans la synagogue (Werner, «Perspectives on Chagall» 14-16) : Leymarie les étudie, selon Werner, comme s'il s'agissait de vitraux médiévaux, où chaque détail est prescrit, alors que Chagall s'est autorisé des libertés que Leymarie ne semble pas prendre en compte; Werner s'interroge sur le bien fondé de la recherche intensive de Leymarie pour justifier par un passage biblique chaque détail des vitraux de

22. « $[\ldots]$ in his own right, with his great merits and also with his shortcomings - that at last America appreciates la Chagallité », (Werner, « Chagall's Jerusalem windows » 547).

23. « Whether Kiryath Hadassah will have a temple of the muses or a temple of the Lord, time will tell» (541).

24. C'est à dessein, et en pensant à la longue et fructueuse amitié et collaboration entre André Malraux et Marc Chagall que nous utilisons le terme « métamorphose ».

25. « artificielle » car il accuse par exemple les vitraux de Rouault de n'être qu'une « adaptation de tableaux et non des créations originales ». Les réalisations de Matisse à Vence sont également critiqué par Leymarie car « étrangères à la tradition authentique du vitrail-peinture » (Leymarie 15).

26. «[...] Les vitraux de Jérusalem, au-delà de la spiritualité judaïque dont ils sont imprégnés, promeuvent, contre l'indigence et la mutilation de notre époque, un humanisme complet, éternel et nouveau » (Leymarie 26). 
Chagall. Il attire ainsi l'attention sur les dangers d'une surinterprétation des intentions de Chagall. L'argument de Werner consiste donc à dire que si l'analyse décontextualisée d'une oeuvre change son sens, et si l'on ne peut plus les considérer différemment que comme des œuvres d'art moderne c'est bien le dessein même de l'édifice qui est annihilé : « perhaps the little synagogue has been transformed into a museum by the very installation of the windows, and whatever worship is held there is purely incidental. If that is so, the purpose of the edifice has been all but completely annihilated [...]» (15). Ce qui fait regretter à Werner que Chagall n'ait rien proposé de plus enrichissant pour illustrer le $49^{\text {ème }}$ chapitre de la Genèse : « [...] one can regret that Chagall had been given nothing more nourishing than this $49^{\text {th }}$ chapter of Genesis » $(15)$.

Si Werner alerte sur les dangers d'une décontextualisation des vitraux scellés dans une synagogue, tout en admettant leur inévitable muséalisation, Pierre Rondot, qui écrit dès octobre 1961 sur les vitraux pour Jérusalem dans la revue jésuite Etudes, ne craint pas d'affirmer la distance que Chagall aurait volontairement prise avec la foi et le culte, pour preuves ses prises de liberté quant au texte biblique : «Comme l'État sur le territoire duquel ils doivent briller, les vitraux de Chagall procèdent en effet d'une inspiration librement interprétée, qui n'implique pas la foi, ni la participation au culte » (Rondot 122). Cela revient à dire que, même couronnant la bimah d'une synagogue, les vitraux, selon Rondot, n'engagent pas la foi ; c'est sur un socle culturel et non cultuel que s'érige la relation entre l'artiste, l'œuvre et le spectateur : en effet, pour Rondot, « les vitraux de Chagall [...] exigent la connaissance, le respect et [...] l'assimilation de la tradition » (122). D'ailleurs, ce sont dans les mêmes termes que Chagall répond à Daniel Barès : « je m'adapte et respecte les traditions en tâchant de trouver encore plus de liberté, si possible » (Panorama chrétien 50), propos qui apparaissent très distanciés de toute considération religieuse en effet.

Les commentaires liés à la scénographie sont également révélateurs de la réception que visiteurs et critiques d'art réservent aux vitraux de Chagall. Nous savons grâce à Werner que la monstration des vitraux à New York étaient très différente de celle de Paris (pas de pavillon dédié, mais une exposition dans les salles habituelles, jusque dans l'escalier du musée). Les visiteurs de l'exposition des vitraux pouvaient donc admirer également les œuvres de Redon. Cela offre à Werner l'occasion de porter un discours sur l'art moderne et les questions de poétique, plaçant d'emblée Chagall en successeur de Redon et Gauguin, autrement dit inscrivant Chagall avant tout dans une généalogie artistique moderne. Par ailleurs, Werner rapporte les réactions des visiteurs découvrant les vitraux dans la synagogue :

« A few visitors have complained that the synagogue was too small for the windows, that they could not be looked at from sufficient a distance, that is to say, from a angle that is not too high. These people 
said that they had to crane their necks to gaze up at the windows » (Werner, " Chagall's Jerusalem windows » 552-53.). ${ }^{27}$

Ce témoignage très concret montre les attentes d'une certaine population de visiteurs, venant davantage admirer les œuvres d'un peintre moderne, que s'imprégner d'objets d'art sacré à vocation cultuelle. Ainsi, le fait même d'exposer en musée des vitraux prévus pour un lieu de culte témoigne de la reconnaissance d'un Chagall moderne, l'amateur d'art qui visite l'exposition étant plus fréquemment motivé par la beauté plastique des œuvres. Ce phénomène s'appliquera de la même manière au cycle des dix-sept toiles prévues pour la chapelle du Calvaire à Vence, et auxquelles sera finalement dédié un musée, voulu, là encore, par Malraux. En 2007 le changement d'appellation du musée sera acté, abandonnant le nom de musée national Message Biblique Marc Chagall au profit du moins biblique musée national Marc Chagall ${ }^{28}$.

Les paramètres abordés dans la réception des deux expositions et du projet final dans la synagogue, le lieu et la scénographie (on pourrait rajouter le public) montrent la transformation qui s'opère entre la nature et la vocation de ces œuvres, et leur réception. En effet, on assiste à une métamorphose de la nature des œuvres, non plus objets cultuels mais objets d'art. Si l'on replace ce qui est, en somme, un succès personnel pour Chagall dans le contexte des années 1960, le fait d'être considéré comme un peintre moderne, alors même qu'il expose des vitraux à thème biblique destinés à un lieu de culte, peut s'expliquer de deux manières : d'une part, durant les années 1950, les grands noms de l'art des premières années du $\mathrm{XX}^{\text {ème }}$ siècle sont déjà morts ${ }^{29}$, notamment les peintres juifs de sa génération ; durant les décennies d'après-guerre, «Picasso, Mirò et Chagall faisaient donc figure de grands anciens » (Wullschläger 476) ; d'autre part la profonde amitié, qui aura duré près de cinquante ans, entre Malraux et Chagall, et qui facilita de fructueuses collaborations entre l'artiste et l'État mécène, a permis de poser une regard décloisonné sur l'œuvre de l'artiste. Si avant les deux expositions temporaires parisienne et newyorkaise des vitraux pour Jérusalem et leur installation sur les murs de la synagogue la communauté juive était souvent critique à l'égard du travail de Chagall incluant l'iconographie chrétienne, ces trois événements s'avèreront l'occasion d'un second réveil de la critique juive au cours de ce XX ${ }^{\text {ème }}$ siècle,

27. Suite de la citation : « But the majority did not seem to mind. On the other hand, the effects achieved in the Museum of Modern Art were rather static and inchangeable due to the problem of installing the windows in the stairhall and the manner of lighting them [...] from behind [...]. In Jerusalem, however, there is a continuous collaboration between art and nature » (553-54).

28. Courrier cosigné par Meret Meyer, Bella Meyer, Piet Meyer et David McNeil, daté du 24 octobre 2007 et adressé à Madame Francine Mariani-Ducray, directrice des Musées de France (archives Comité Chagall).

29. Voir à ce sujet Jackie Wullschläger. 
après celui des années $1920^{30}$, tandis que la critique d'art contemporain, autrefois très divisée et somme toute peu bavarde sur l'œuvre et sur l'artiste, admet désormais Chagall pleinement et en connaissance de cause dans le cercle des Grands Maîtres de l'art moderne.

En faisant appel à un artiste de renommée mondiale, on constate donc que le regard porté sur les douze vitraux dans la synagogue se trouve déjà en partie modifié, métamorphosant le cycle à vocation cultuel, par son emplacement, en un programme artistique. Le point de vue juif est également renouvelé puisque Chagall est désormais pleinement reconnu comme le plus grand peintre juif, quoique souvent considéré en marge des autres artistes juifs, point de vue rendu possible précisément grâce à cette nouvelle considération de son œuvre comme majeure dans l'art contemporain : certains de ses co-religionnaires y voient, au-delà de l'acceptation du peintre juif dans le cercle restreint des grands maîtres de l'art moderne, la reconnaissance de l'existence même d'un art juif.

Ainsi, ce tour d'horizon muséographique a permis d'appréhender la modification sémantique qui s'est opérée entre le projet et la réalisation des vitraux dans la synagogue, et leur exposition dans deux musées d'art moderne. D'objets cultuels par essence, les vitraux deviennent immanquablement objets d'art. Mais la singularité des vitraux commandés à Chagall réside davantage dans le fait que, du fait du statut et de la renommée de l'artiste judéo-russe devenu français, c'est dès la genèse du projet que les douze vitraux revêtent une portée politique, délaissant malgré eux leur valeur intrinsèquement religieuse. Loin d'être fortuit, le choix de Miriam Freund, autant que le contexte artistique autour des édifices religieux, juifs ou chrétiens, dans lequel s'inscrit ce choix, témoigne d'une démarche parfaitement avisée.

Pourtant, cette approche était bien éloignée de Chagall lui-même. Si ces différents séjours en Palestine ${ }^{31}$ l'ont particulièrement marqué, éveillant chez lui le sentiment de fouler la terre de ces ancêtres et de cheminer à l'intérieur de la Bible, l'artiste n'a jamais revendiqué aucune idée à prendre stricto sensu d'un point de vue politique. Au contraire, les vitraux pour Jérusalem, comme les autres d'ailleurs, sont un pas-de-deux où la créature cherche le Créateur, où le plasticien révèle le Démiurge : « regardez! J'ai tout les atouts avec moi : la lumière c'est celle du ciel, [...] ! le feu [...] vient aussi du ciel $[\ldots]$. J'ai la matière. [...] si ce que j'ai fait tient à côté de la matière, alors j'ai gagné. [...] parce qu'il y a Dieu derrière $)^{32}$.

30. A Paris. Voir à ce sujet Jarrassé, (Existe-t-il un art juif? 2006).

31. Chagall s'était déjà rendu trois fois en Palestine avant le projet pour la synagogue : en 1931 (pour préparer les gravures pour la Bible), en 1951 (pour une grande exposition de ses œuvres) et en 1957.

32. Propos recueillis par Pierre Cabanne (47-48). 
On assistera, moins d'un an plus tard, à une sorte de chassé-croisé de la réception des œuvres cultuels et culturelles de Chagall : après avoir été encensé, dans le paysage artistique français et international, comme l'un des plus grands peintres modernes réalisant un véritable joyau de l'art du vitrail à vocation cultuelle, il sera lourdement décrié lorsque son ami Malraux rendra public la commande d'un nouveau décor pour le plafond d'un édifice aussi emblématique dans le patrimoine français que l'Opéra Garnier. Il faudra attendre l'inauguration du plafond pour que les critiques s'avisent de leur méprise et louent l'œuvre de Chagall.

\section{OUVRAGES CITÉS}

AZRIA, Régine. « La visibilité du judaïsme dans la France contemporaine : vitalité ou quiproquo ?». Autres Temps. Cahiers d'éthique sociale et politique 69 (2001) : 16-30.

BARAM, Sioma. « Chagall. Vitraux pour Jérusalem ». L’Arche 55-56 (1961) : 106.

BOURDET, Denise. « Marc Chagall ». La Revue de Paris (août 1961) : 141-146.

BOUVIER, Yves et Cousin CHRISTOPHE. Audincourt. Le sacre de la couleur. Fernand Léger, Jean Bazaine, Maurice Novarina, Jean Le Moal au Sacré-Cœur. CRDP de Franche-Comté : Néo Éditions, 2007.

CABANNE, Pierre. «Chagall rend la lumière sa liberté ». Chagall. Vitraux pour Jérusalem. Musée des Arts Décoratifs (Paris, 16 juin - 30 septembre 1961). Paris : Musée des Arts décoratifs, 1961 : 40-48.

Chagall. Vitraux pour Jérusalem. Musée des Arts Décoratifs (Paris, 16 juin - 30 septembre 1961). Paris : Musée des Arts décoratifs, 1961.

CHARENSOL, Georges. «Les vitraux de Chagall ». Revue des deux Mondes (1 $1^{\text {er }}$ août 1961) : 484-486.

CHARTIER, Roger. Au bord de la falaise. 1998. Paris : Albin Michel, 2009.

«Entretien avec Chagall». Propos recueillis par Marcelle Berr de Turique. L'Arche 55-56 (1961) : 82-87 et 115.

FREUND, Miriam. Jewels for A Crown: The Story of the Chagall Windows. New York : McGraw-Hill, 1963.

—. In my Lifetime: Family, Community, Zion. New York : Town House Press, 1989.

HARSHAV, Benjamin. Chagall and His Times. Stanford : Stanford University Press, 2004.

—. «Les vitraux de Jérusalem à la lumière de la poétique chagallienne ». Marc Chagall. Hadassah, de l'esquisse au vitrail. Paris : Musée d'art et d'histoire du Judaïsme, 2002 : 119-129.

JARRASSÉ, Dominique. Existe-t-il un art juif? Paris : Éditions Biro, 2006. 
—. « Remarques sur la symbolique des vitraux de Jérusalem ». Chagall et le vitrail. De la pierre à la lumière. Clermont-Ferrand : musée des Beaux-Arts, 1993. Catalogue d'exposition (Clermont-Ferrand, Musée des beaux-arts, juin - septembre 1993). 46-49.

—. Une Histoire des synagogues françaises. Entre Occident et Orient. Paris : Actes Sud, 1997.

KRIGIER, Rivon. «La disposition des tribus dans les vitraux de Chagall ». Marc Chagall. Hadassah, de l'esquisse au vitrail. Paris : Musée d'art et d'histoire du Judaïsme, 2002 : 20-21.

L’Arche, revue du FNJU, n55-56, août-septembre 1961.

LEYMARIE, Jean. Vitraux pour Jerusalem, Paris : Éditions A. Sauret, 1962.

Marc Chagall. Hadassah, de l'esquisse au vitrail. Catalogue d'exposition (Paris, Musée d'art et d'histoire du Judaïsme, 30 avril-15 septembre 2002). Paris : Musée d'art et d'histoire du Judaïsme ; Adam Biro, 2002.

« Marc Chagall vous présente ses vitraux pour Jérusalem ». Panorama chrétien 55 (décembre 1961) : 47-50.

PERL, Jed. «The Spiritual in Art». New Republic (18 février 2009). URL : https://newrepublic.com/article/61380/ the-spiritual-art (page consultée le 20 mai 2019).

ROGER-MARX, Claude. « Les vitraux de Chagall ». La Revue de Paris (août 1961) : 157-158.

RONDOT, Pierre. «Vitraux de Chagall pour Jerusalem ». Études (octobre 1961) : 122-123.

Un art d'État? Commandes publiques aux artistes plasticiens, 1945-1965. Catalogue d'exposition (Pierrefittesur-Seine, Archives nationales, 30 mars - 13 juillet 2017). Rennes : Presse Universitaire de Rennes, 2017.

WERNER, Alfred. «Perspectives on Chagall ». Congress Bi-Weekly 29:18 (24 décembre 1962) : 14-16.

—. "Chagall's Jerusalem Windows ». No Graven Images: Studies in Art and the Hebrew Bible. Ed. Joseph Gutmann. New York: Ktav, 1971. 527-555. [Première édition dans Art Journal (1962) : 224-232].

WASSERSTEIN, Bernard. Les Juifs d'Europe depuis 1945. Paris : Calmann-Lévy, 2000.

WULLSCHLÄGER, Jackie. Chagall. Paris : Gallimard, 2008.

Résumé : À l'été 196I, Marc Chagall expose à Paris douze vitraux conçus pour les quatre murs de la synagogue du Centre médical Hadassah à Jérusalem. La majorité de ces douze vitraux sera ensuite exposée à New York à l'automne de la même année, avant que le cycle au complet ne soit définitivement scellé et inauguré à Jérusalem en février 1962. À cette époque de sa carrière, Chagall a déjà réalisé deux vitraux pour Assy (I956-I957), et, en I958, il commence sa fructueuse collaboration avec Charles Marq pour la réalisation des vitraux de la cathédrale de Metz qui l'occupera jusqu'en 1968. Cet article propose d'interroger la singularité chez Chagall d'exposer des vitraux dans des musées d'art. En effet, cette singularité est doublement notable : par leur nature monumentale, les vitraux ne sont pas des objets d'expositions temporaires; par leur vocation première à décorer un lieu de 
culte, une modification sémantique s'opère sur l'objet, à partir du moment où il est présenté dans un musée. C'est cette modification sémantique et ses conséquences sur la réception de l'artiste qui sont abordées ici.

Abstract: In the summer of 1961, Marc Chagall exhibited in Paris twelve stained glass windows designed for the four walls of the Hadassah Medical Center synagogue in Jerusalem. The majority of these twelve stained glass windows were then exhibited in New York in the autumn of the same year, before the completion of the entire cycle, inaugurated in Jerusalem in February 1962. At this period of his career, Chagall had already achieved two stained glass windows for Assy (1956-1957), and in 1958 he began his fruitful collaboration with Charles Marq for the realization of the stained glass windows of the Metz cathedral, which will keep him busy until 1968. In this article, Chagall's singularity of exhibiting stained glass in art museums is considered. Indeed, this singularity is doubly significant: by their monumental nature, the stained glass windows are not objects of temporary exhibitions; by their vocation to decorate a place of worship, a semantic modification takes place around the object, from the moment it is displayed in museums. It is this semantic modification that is being discussed here, and its consequences on the reception of the artist and his works. 